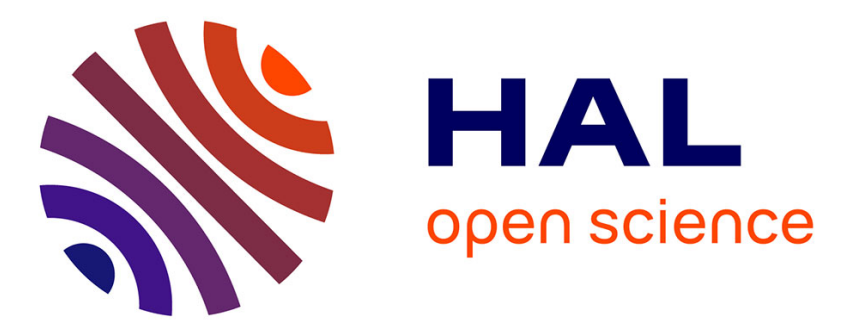

\title{
Maximum entropy modeling of discrete uncertain properties with application to friction
}

R. Murthy, B.-K. Choi, X. Q. Wang, M. C. Sipperley, M. P. Mignolet, Christian Soize

\section{- To cite this version:}

R. Murthy, B.-K. Choi, X. Q. Wang, M. C. Sipperley, M. P. Mignolet, et al.. Maximum entropy modeling of discrete uncertain properties with application to friction. Probabilistic Engineering Mechanics, 2016, 44, pp.128-137. 10.1016/j.probengmech.2015.10.003 . hal-01276290

\section{HAL Id: hal-01276290 \\ https://hal.science/hal-01276290}

Submitted on 19 Feb 2016

HAL is a multi-disciplinary open access archive for the deposit and dissemination of scientific research documents, whether they are published or not. The documents may come from teaching and research institutions in France or abroad, or from public or private research centers.
L'archive ouverte pluridisciplinaire HAL, est destinée au dépôt et à la diffusion de documents scientifiques de niveau recherche, publiés ou non, émanant des établissements d'enseignement et de recherche français ou étrangers, des laboratoires publics ou privés. 


\title{
MAXIMUM ENTROPY MODELING OF DISCRETE UNCERTAIN PROPERTIES WITH APPLICATION TO FRICTION
}

\author{
RAGHAVENDRA MURTHY ${ }^{1}$, BYEONG-KEUN CHOI ${ }^{1,2}$, X.Q. WANG ${ }^{1}$, \\ MARK C. SIPPERLEY ${ }^{1}$, MARC P. MIGNOLET ${ }^{1}$, and CHRISTIAN SOIZE ${ }^{3}$ \\ ${ }^{1}$ SEMTE, Faculties of Mechanical and Aerospace Engineering, Arizona State University, \\ Tempe AZ 85287-6106, USA \\ E-mails:rnmurthy@asu.edu,bgchoi@asu.edu,xiaoquan.wang.1@asu.edu, \\ mark.sipperley@asu.edu,marc.mignolet@asu.edu. \\ ${ }^{2}$ Gyeongsang National University, Department of Energy and Mechanical Engineering, \\ Institute of Marine Industry, Tongyoung, Gyeongnam 650-160, South Korea. \\ ${ }^{3}$ Laboratoire Modélisation et Simulation Multi Echelle, Université Paris-Est, 5, Bd \\ Descartes,77454 Marne-la-Vallée Cedex 02, France. \\ E-mail: christian.soize@univ-paris-est.fr
}

\begin{abstract}
The first part of the present investigation focuses on the formulation of a novel stochastic model of uncertain properties of media homogenous in the mean which are represented as stationary processes. In keeping with standard spatial discretization methods (e.g., finite elements), the process is discrete. It is further required to exhibit a specified mean, standard deviation, and a global measure of correlation, i.e., correlation length. The specification of the random process is completed by imposing that it yields a maximum of the entropy. If no constraint on the sign of the process exists, the maximum entropy is achieved for a Gaussian process the autocovariance of which is constructed. The case of a positive process is considered next and an algorithm is formulated to simulate the non-Gaussian process yielding the maximum entropy.

In the second part of the paper, this non-Gaussian model is used to represent the uncertain friction coefficient in a simple, lumped mass model of an elastic structure resting on a frictional support. The dynamic response of this uncertain system to a random excitation at its end is studied, focusing in particular on the occurrence of slip and stick.
\end{abstract}

Keywords: Friction, uncertainty, microslip, stochastic process, maximum entropy, simulation.

\section{Introduction}

The modeling of uncertain geometric and/or material properties as random variables, stochastic processes, or random fields has received significant attention in the last 2 decades, e.g., see Ghanem and Spanos (1991), Schueller (2007), Sachdeva et al. (2007) and Soize (2012) for discussion and some methods review. A key challenge in using such models in practical situation is the often dramatic lack of information available on the uncertainty. More specifically, the mean value of the geometric/material property considered is generally believed to be known from past experience and/or available data. However, even the level of uncertainty, e.g., standard deviation, is often less clearly known and may in fact be considered as a variable in a 
parametric study. Sometimes, an upper and/or lower bound may also be known because of an acceptance/rejection test carried out on all samples. However, more detailed information is very often not available.

This limited data does not permit the analyst to build a specific probabilistic model who is then forced to make additional assumptions. This perspective has led Soize $(2000,2001)$ to propose that the stochastic description of the uncertainty model be derived by maximizing the statistical entropy under constraints representing the true knowledge on the stochastic model. The maximization of the entropy induces a maximum spread of the uncertainty (consistently with the constraints) in the tail of the distribution and thus to the consideration of "wide-spread" uncertainty that provides a good perspective on the effects of variations of the system properties, even those that are "far" from the mean. Thus, this approach, also referred to as the nonparametric stochastic modeling approach, requires only partial knowledge of the system uncertainty complemented by the single assumption of maximization of entropy.

In its original formulation (Soize, 2000, 2001), the nonparametric stochastic modeling approach was used for characterization of the mass, damping, and stiffness matrices of reduced order/modal models, not to the detailed modeling of any specific property such as mass density or Young's modulus. Such a characterization was carried out in later extension (Soize, 2006) to matrix-valued fields focusing in particular on the modeling of the elasticity tensor of random media.

The present effort complement this work by addressing the modeling of scalar random properties, of variable or constant sign, of media homogenous in the mean as stationary processes. In keeping with standard spatial discretization methods (e.g., finite elements), the process is discrete. The proposed modeling relies on the specification of only the mean and standard deviation of the property as well as on a global measure of the correlation, i.e., a correlation length. The maximization of the entropy then provides the description of the process consistent with this prescribed information.

The above stochastic modeling technique will be exemplified here on a property seldom considered and yet exhibiting well known uncertainty, i.e., friction. When two deformable bodies are in extended contact with each other, as in joints, turbomachinery blade friction dampers, brakes, etc., the coefficient of friction between them must be defined over the contact zone, i.e., as a spatially varying property which governs the occurrence of stick, microslip, or macroslip (e.g., see Sinha and Griffin, 1985, Berger and Deshmukh, 2005, Deshmukh et al., 2007, Segalman et al., 2009, and references therein). After an appropriate spatial discretization of the contact zone, it becomes then necessary to specify the coefficient of friction at a set of discrete locations. The uncertainty in the values of this coefficients resulting from unknown spatial variations of roughness, temperature, composition, etc. then calls for the stochastic modeling considered in the first part of the paper. To demonstrate this application and presents a first perspective in this problem, a simple dynamic model of this contact problem is adopted here as a cascade of 5 oscillators with Jenkins friction elements. The effects of uncertainty in the coefficients of friction on the dynamic response of this system is then studied.

\section{Maximum Entropy Discrete Process}

\subsection{General Derivation}

Let $X_{n}$ denote a discrete stationary process defined over the domain $n \in I=\{l, l+1, \ldots, u\}$ and define the random vector $\underline{X}=\left[X_{l} X_{l+1} \ldots X_{u}\right]^{T}$ where ${ }^{T}$ denotes the operation of matrix/vector transposition. Then, the entropy $S$ of $\underline{X}$ is defined as 


$$
S \equiv-E\left[\ln \left(p_{\underline{X}}(\underline{x})\right)\right]=-\int_{\Omega} \ln \left(p_{\underline{X}}(\underline{x})\right) p_{\underline{X}}(\underline{x}) d \underline{x}
$$

where $E[$.$] denotes the operation of$ mathematical expectation and $\Omega$ is the domain of support of the values of the process. If no signature constraint is enforced, both positive and negative values of the process are allowed and thus

$$
\begin{aligned}
\Omega= & \left\{\left(x_{l}, x_{l+1}, \ldots, x_{u}\right) \in\right. \\
& (-\infty, \infty) \times(-\infty, \infty) \ldots \times(-\infty, \infty)\} .
\end{aligned}
$$

If a positive sign of the process is required,

$$
\begin{array}{r}
\Omega=\left\{\left(x_{l}, x_{l+1}, \ldots, x_{u}\right) \in\right. \\
[0, \infty) \times[0, \infty) \ldots \times[0, \infty)\} .
\end{array}
$$

In Eq. (1), $p_{\underline{X}}(\underline{x})$ denotes the probability density function of the random vector $\underline{X}$ evaluated at a realization point $\underline{x}$. Since $X_{n}$ is stationary, its joint probability density functions satisfy the usual independence under a uniform shift along $I$, e.g.,

$$
p_{X_{n}}(x)=p_{X_{n+1}}(x)
$$

and $\quad p_{X_{n} X_{m}}(x, y)=p_{X_{n+1} X_{m+1}}(x, y)$.

It is desired here to determine the probability density function $p_{X}(\underline{x})$ which maximizes the entropy, Eq. (1), under the constraints that:

(i) the total probability is one, i.e.,

$$
\int_{\Omega} p_{\underline{X}}(x) d \underline{x}=1
$$

(ii) the mean and variance are given and constant (since the process is stationary)

$$
\begin{aligned}
\int_{\Omega} x_{n} p_{\underline{X}}(\underline{x}) d \underline{x}=\mu_{X} \quad n \in I \\
\int_{\Omega}\left(x_{n}-\mu_{X}\right)^{2} p_{\underline{X}}(\underline{x}) d \underline{x}=\sigma_{X}^{2} \quad \text { for } n \in I
\end{aligned}
$$

(iii) a correlation length is given. Two such measures are (Soize, 2006, Nigam, 1983)

$$
L_{0}=\sum_{m=1}^{\infty}\left|K_{X X}(m)\right| / K_{X X}(0)
$$

and $\quad L_{1}=\sum_{m=0}^{\infty} m\left|K_{X X}(m)\right| / \sum_{m=0}^{\infty}\left|K_{X X}(m)\right|$ where $K_{X X}(m)$ is the stationary autocovariance function. It is defined as

$K_{X X}(m)=\Gamma_{X X}(n, n+m)$ for any $n \in I$ (10a) where

$$
\begin{aligned}
& \Gamma_{X X}(n, n+m)=E\left[\left(X_{n}-\mu_{X}\right)\left(X_{n+m}-\mu_{X}\right)\right] \\
& =\int_{\Omega}\left(x_{n}-\mu_{X}\right)\left(x_{n+m}-\mu_{X}\right) p_{\underline{X}}(\underline{x}) d \underline{x}
\end{aligned}
$$

The series involved in Eqs (8) and (9) will be truncated to finite sum for $m=0$ to $m_{\max }$ and thus can be written as

$$
\begin{aligned}
& \sum_{m=0}^{m_{\max }} a_{m} s_{m} \Gamma_{X X}(n, n+m)=0 \text { for any } n \\
& \text { where } \quad s_{m}=\operatorname{sgn}\left(K_{X X}(m)\right) \\
& \qquad a_{m}=\left(1+L_{0}\right) \delta_{m 0}-1 \quad m \in\left[0, m_{\max }\right],
\end{aligned}
$$

with $\delta_{i j}$ the Kronecker symbol, for the correlation length $L_{0}$ while for $L_{1}$

$$
a_{m}=L_{1}-m \quad m \in\left[0, m_{\max }\right] .
$$

For the optimization of the entropy, Eqs (5)-(7), (11) can be written in the generic form

$$
\Xi_{\text {in }} \equiv \int_{\Omega} f_{\text {in }}(\underline{x}) p_{\underline{X}}(\underline{x}) d \underline{x}-C_{i}=0
$$

where $\quad f_{1 n}\left(x_{n}\right)=x_{n}-\mu_{X}$

$$
f_{2 n}(\underline{x})=\sum_{m=0}^{m_{\max }} a_{m} s_{m}\left(x_{n}-\mu_{X}\right)\left(x_{n+m}-\mu_{X}\right)
$$

$f_{3 n}(\underline{x})=\left(x_{n}-\mu_{X}\right)^{2}$ and $f_{4}=1$

with $C_{1}=C_{2}=0, C_{3}=\sigma_{X}^{2}$, and $C_{4}=1$.

Then, the maximization of the entropy, Eq. (1) under the constraints of Eqs (15)-(19) can be accomplished in the Lagrange multiplier framework and yields

$$
\begin{gathered}
p_{\underline{X}}(\underline{x})=\frac{1}{e} \exp \left[-\sum_{n} \lambda_{1 n} f_{1 n}-\sum_{n} \lambda_{2 n} f_{2 n}\right. \\
\left.-\sum_{n} \lambda_{3 n} f_{3 n}-\lambda_{4} f_{4}\right] \quad \underline{x} \in \Omega
\end{gathered}
$$

where $\lambda_{1 n}, \lambda_{2 n}, \lambda_{3 n}$, and $\lambda_{4}$ are the Lagrange multipliers associated with the 
constraints of Eqs (16)-(19). Next, note that the term in bracket is a quadratic form of the vector $\underline{x}-\mu_{X} \underline{E}$, where $\underline{E}$ is the vector whose components are all equal to 1 , i.e., $\underline{E}=[\ldots 111 \ldots]^{T}$. Thus, the above probability density function can be rewritten as

$$
\begin{aligned}
p_{\underline{X}}(\underline{x})= & C^{\prime} \exp \left[-\frac{1}{2}\left(\underline{x}-\mu_{X} \underline{E}\right)^{T} G^{\prime}\left(\underline{x}-\mu_{X} \underline{E}\right)\right. \\
& \left.-\underline{V}^{T}\left(\underline{x}-\mu_{X} \underline{E}\right)\right] \quad \underline{x} \in \Omega
\end{aligned}
$$

where

$$
C^{\prime}=\exp \left(-\lambda_{4}-1\right)
$$

and the elements of the matrix $G^{\prime}$ and vector $V$ are

$$
G_{n(n+m)}^{\prime}=2 \lambda_{2 n}\left[a_{m} s_{m}\right]+2 \lambda_{3 n} \delta_{m 0}
$$

for $m \in\left[0, m_{\max }\right]$ and 0 otherwise, and

$$
V_{n}=\lambda_{1 n} .
$$

Assuming that the matrix $G^{\prime}$ is not singular,

Eq. (21) can finally be rewritten in the familiar form

$$
p_{\underline{X}}(\underline{x})=C \exp \left[-\frac{1}{2}(\underline{x}-\underline{\mu})^{T} G(\underline{x}-\underline{\mu})\right]
$$

for $\underline{x} \in \Omega$ where

$$
\begin{gathered}
C=C^{\prime} \exp \left[\frac{1}{2} \underline{V}^{T} G^{-1} \underline{V}\right] \\
\underline{\mu}=\mu_{X} \underline{E}-G^{-1} \underline{V} \\
G=\frac{1}{2}\left(G^{\prime}+G^{\prime T}\right) .
\end{gathered}
$$

To complete the characterization of the distribution of Eq. (25), it remains to evaluate the parameters it involves, i.e., the Lagrange multipliers $\lambda_{1 n}, \lambda_{2 n}, \lambda_{3 n}$, and $\lambda_{4}$, from the constraints they represent, i.e., Eqs (5), (6), (11), and (15). In that regard, note that it is more convenient to directly focus on the evaluation of $C, \underline{\mu}, \lambda_{2 n}$, and $\lambda_{3 n}$ the latter two defining the matrix $G$.

Of special interest here is the situation in which the size of the domain $I$ becomes large, i.e., $l \rightarrow-\infty$ and $u \rightarrow \infty$. Then, each random variable $X_{n}$ exhibits the same properties, none of them being closer or further from the boundaries $l$ and $u$ of the domain $I$. Thus, the dependence of $p_{\underline{X}}(\underline{x})$ on any variable $x_{n}$ should be the same which occurs when $\underline{\mu}=\mu \underline{E}, \quad \lambda_{2 n}=\lambda_{2}$ and $\lambda_{3 n}=\lambda_{3}$ (29a)-(29c) for which the matrix $G$ is Toeplitz and symmetric. With Eqs (29b) and (29c), the matrix $G$ becomes

$$
G_{n(n \pm m)}=\left(\lambda_{3}\right)\left\{2 \delta_{m 0}+\delta a_{m} s_{m}\right\}
$$

for $m \in\left[0, m_{\max }\right]$ and where the parameter $\delta=\lambda_{2} / \lambda_{3}$ is introduced in place of $\lambda_{2}$. Note that the signs $s_{m}= \pm 1$ are dependent on the autocovariance function, see Eq. (12), and thus are unknown at this point.

Equations (25), (29a), and (30) represent the maximum entropy distribution sought. It remains however to address the determination of the parameters $C, \mu, \lambda_{3}, s_{m}$, and $\delta$ from Eqs (5), (6), (7), and (11) and the efficient simulation of realization of the random values $X_{n}$. These issues are addressed in the ensuing sections.

\subsection{Process Without Sign Constraint - Direct Estimation of the Autocovariance Function}

The case of a process without sign constraint, i.e., with the domain $\Omega$ defined by Eq. (2) is considered first because of its simplicity. Under this assumption, it is concluded from Eq. (25) that the random vector $\underline{X}$ is Gaussian with mean $\mu$ and with covariance matrix

$$
\hat{K}_{\underline{X} \underline{X}}=G^{-1} \text {. }
$$

Note that $\hat{K}_{\underline{X} X}$ is expected to exhibit a Toeplitz structure, as $G$ does, owing to the stationarity of the process in its autocovariance function.

To satisfy the constraints of Eqs (5) and (6), one obtains directly 


$$
C=\sqrt{\frac{\operatorname{det}(G)}{(2 \pi)^{P}}}
$$

where $P$ denotes the number of values of the process considered and

$$
\mu=\mu_{X} \text {. }
$$

Next, consider the parameter $\lambda_{3}$ and note that it scales (for a fixed value of $\delta$ ) the matrices $G$ and $\hat{K}_{\underline{X}} \underline{X}$. Its effects are thus (i) to uniformly scale the values of the process, more specifically by the factor $1 / \sqrt{\left|\lambda_{3}\right|}$, and (ii) to modify the sign of the autocovariance function. Thus, the parameter $\lambda_{3}$ does not affect the correlation length which is then only a function of $\delta$ and of the selected sign sequence $s_{m}$ (see Eq. (12a)). Once these quantities have been determined from either Eq. (8) or (9) and Eq. (12a), i.e., by solving

$$
L_{0}\left(\delta, s_{m}\right)=\bar{L}_{0} \quad \text { or } \quad L_{1}\left(\delta, s_{m}\right)=\bar{L}_{1}
$$

where $\bar{L}_{0}$ or $\bar{L}_{1}$ is the imposed correlation length, the value of $\lambda_{3}$ can then be selected to match the variance condition, Eq. (7).

The numerical evaluation of $L_{0}\left(\delta, s_{m}\right)$ and $L_{1}\left(\delta, s_{m}\right)$ for specified values of $m_{\max }, \delta$, and of the sign sequence $s_{m}$ was achieved as follows. The matrix $G / \lambda_{3}$ was first formed for $P=2 * M+1$ random variables $X_{n}$. The parameter $M$ was selected "large enough", i.e., much larger than $m_{\max }$ for the inverse $\left(G / \lambda_{2}\right)^{-1}=\tilde{K}_{X X} \quad$ to exhibit a near Toeplitz structure. Since the convergence to this structure occurred faster for the elements near the center of the matrix, the $M+1$ st row was considered representative of the true infinite Toeplitz matrix and was used for the estimation of the autocovariance values as

$$
K_{X X}(m)=\frac{1}{\lambda_{3}}\left(\tilde{K}_{\underline{X}} \underline{X}\right)_{M+1, M+1+m} .
$$

Since the variance $K_{X X}(0)$ must be positive, the sign of $\lambda_{3}$ was selected as $\operatorname{sgn}\left(\lambda_{3}\right)=\operatorname{sgn}\left[\left(\tilde{K}_{\underline{X}}\right)_{M+1, M+1}\right]$. Then, the correlation length, $L_{0}\left(\delta, s_{m}\right)$ or $L_{1}\left(\delta, s_{m}\right)$, and the sign sequence $\operatorname{sgn}\left(K_{X X}(m)\right)$ were estimated and compared to $\bar{L}_{0}$ or $\bar{L}_{1}$ and $s_{m}$. A match of both indicated that acceptable values $\delta$ and $S_{m}$ were found. Next, the magnitude of $\lambda_{3}$, was determined to satisfy the variance normalization condition, Eq. (7). Finally, the constants $\mu$ and $C$ were obtained from Eqs (32) and (33) completing the determination of the probability density function.

Multiple solutions (acceptable values $\delta$ and $s_{m}$ ) were occasionally found. In such cases, the solution yielding the highest value of the entropy was retained. In this regard, introducing the expression of the probability density function given by Eq. (26) in Eq. (1) yields

$$
S=-\ln C+\frac{1}{2} E\left[(\underline{X}-\underline{\mu})^{T} G(\underline{X}-\underline{\mu})\right]
$$

or, using Eq. (32),

$$
S=-\ln C+\frac{P}{2} .
$$

The simulation of random values $X_{n}$ can be achieved by standard algorithms. In particular, samples of the random vector $\underline{X}$ can be obtained from the relation

$$
\underline{X}=\underline{\mu}+L \underline{W}
$$

where $L$ is the Cholesky decomposition of $G^{-1}$, i.e., $G^{-1}=L L^{T}$, and the components of the vector $\underline{W}$ are independent zero mean and unit variance Gaussian random variables.

The approach described above was applied with the 2 definitions of the correlation length, Eqs (8) and (9), for $\bar{L}_{0}$ or $\bar{L}_{1}=0.3$, and for several values of $m_{\max }$, see Fig. 1 .

It appears from these figures (note the logarithmic scale) that the autocovariance 
function values for lags $m \geq 1$ are steadily decreasing as $m_{\max }$ increases suggesting that the process converges to a "near white noise" as $m_{\max } \rightarrow \infty$. To satisfy the nonzero correlation length condition, the optimum autocovariance function for large $m_{\max }$ exhibits a very small value (constant/growing linearly for the $L_{0}$ and $L_{1}$ definitions) in the domain $m \in\left[1, m_{\max }\right]$.

Similar findings were also observed with higher correlation lengths (1.0 and 3.0) but the results are not shown here for brevity.
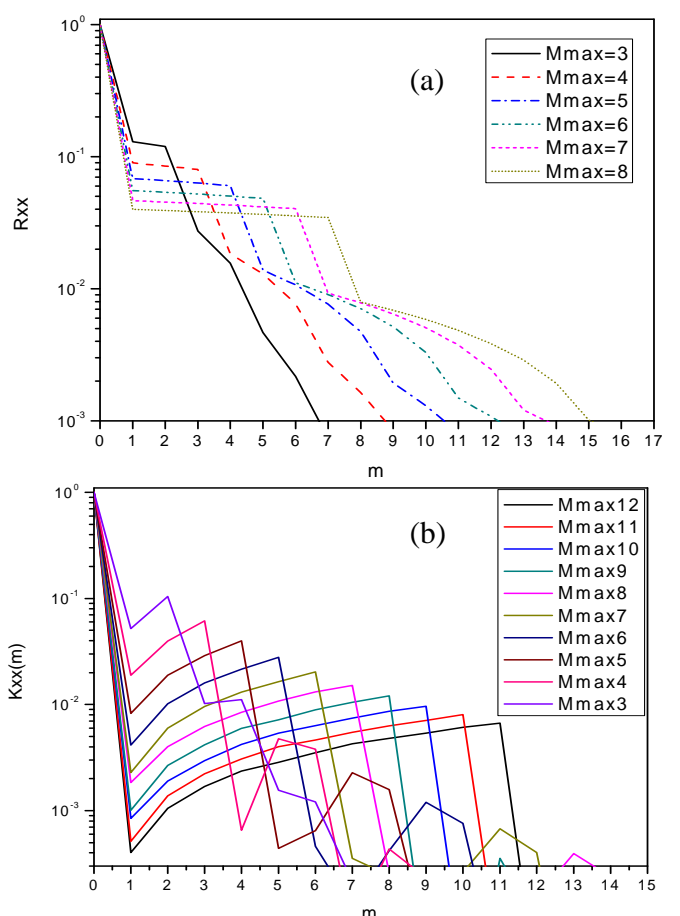

Figure 1. Autocovariance functions obtained for $\sigma_{X}=1$, various $m_{\max }$. (a) $\bar{L}_{0}=0.3$, (b) $\bar{L}_{1}=0.3$.

\subsection{Process Without Sign Constraint - Autoregressive Modeling}

The near white noise behavior of the above solutions is not expected of the physical properties modeled here; it is a reflection of the weakness of the correlation length constraint. To achieve an increased smoothness of the autocovariance function, it is proposed here to adopt a parametric representation for it and/or the process $X_{n}$. An autoregressive modeling is selected for this task as it is known to provide a close representation of a very broad class of autocovariance functions. Specifically, $Z_{n}$ is an autoregressive process of order $M$ when its samples can be recursively computed as

$$
Z_{n}=\hat{b}_{0} W_{n}-\sum_{q=1}^{M} \hat{a}_{q} Z_{n-q}
$$

for $n=M+1, M+2, \ldots$ and in which $W_{n}$ denote independent identically distributed zero mean and unit variance Gaussian random variables and $\hat{a}_{q}$ and $\hat{b}_{0}$ are deterministic coefficients. The process $Z_{n}$ exhibits a stationary behavior when its poles, i.e., the complex roots $v$ of the polynomial

$$
1+\sum_{q=1}^{M} \hat{a}_{q} v^{-q}=0
$$

are less than 1 in magnitude. Its stationary autocovariance function values $K_{Z Z}(m)$ can then be determined from the coefficients $\hat{a}_{q}$ and $\hat{b}_{0}$ through the Yule-Walker equations (e.g., Mignolet and Spanos, 1987)

$$
K_{Z Z}(s)+\sum_{q=1}^{M} \hat{a}_{q} K_{Z Z}(s-q)=\hat{b}_{0}^{2} \delta_{s 0} s \geq 0 .
$$

Specifically, the autocovariance values $K_{Z Z}(m), m=0, \ldots, M$, are first determined by solving the linear system of equations corresponding to Eq. (41) for $s=0, \ldots, M$. Then, the values $K_{Z Z}(m), m>M$ are evaluated recursively from Eq. (41) for $s>M$. The transformation $X_{n}=Z_{n}+\mu_{X}$ leads to the process $X_{n}$ exhibiting the desired mean and the autocovariance function $K_{X X}(m)=K_{Z Z}(m)$ parametrized by the 
coefficients $\hat{a}_{q}$ and $\hat{b}_{0}$. Then, these coefficients will be selected to maximize the entropy, Eq. (37), while satisfying the correlation length and variance constraints, Eq. (7)-(9). Note that the optimization ought to be constrained so that the poles $v$ of the autoregressive model, see Eq. (30), are within the unit circle in the complex plane. However, this constrained was not found to be active in the present investigation.

This autoregressive based optimization approach is typically computationally much less demanding than the direct estimation strategy described in the previous section. The higher cost of the latter method is due to the need to search through the ensemble of combinations of $s_{m}= \pm 1$ for $m=1, \ldots, m_{\max }$ which grows exponentially with $m_{\max }$, or equivalently the correlation length.

The application of the autoregressive modeling approach for $\bar{L}_{0}$ or $\bar{L}_{1}=0.3$, see Fig. 2 , demonstrates that this optimization strategy also leads to near white noise solutions as the autoregressive order $M$ is increased. This result should be fully expected as the increase of the autoregressive order permits a more flexible modeling of the features of the autocovariance function. This unusual convergence process renders difficult the selection of a particular autoregressive model order. A first perspective on this value can be drawn from the corresponding values of the resulting entropy, see Fig. 3, which is bounded on the high side by the entropy of the white noise solution

$$
S_{\max }=\frac{P}{2}\left[1+\ln \left(2 \pi \sigma_{X}^{2}\right)\right] .
$$

It is seen from Fig. 3 that the autoregressive order needed to reach a particular entropy increases with increasing correlation length and is higher for the $\bar{L}_{0}$ definition than for the $\bar{L}_{1}$ one.

A second criterion for the selection of the autoregressive order can be developed from
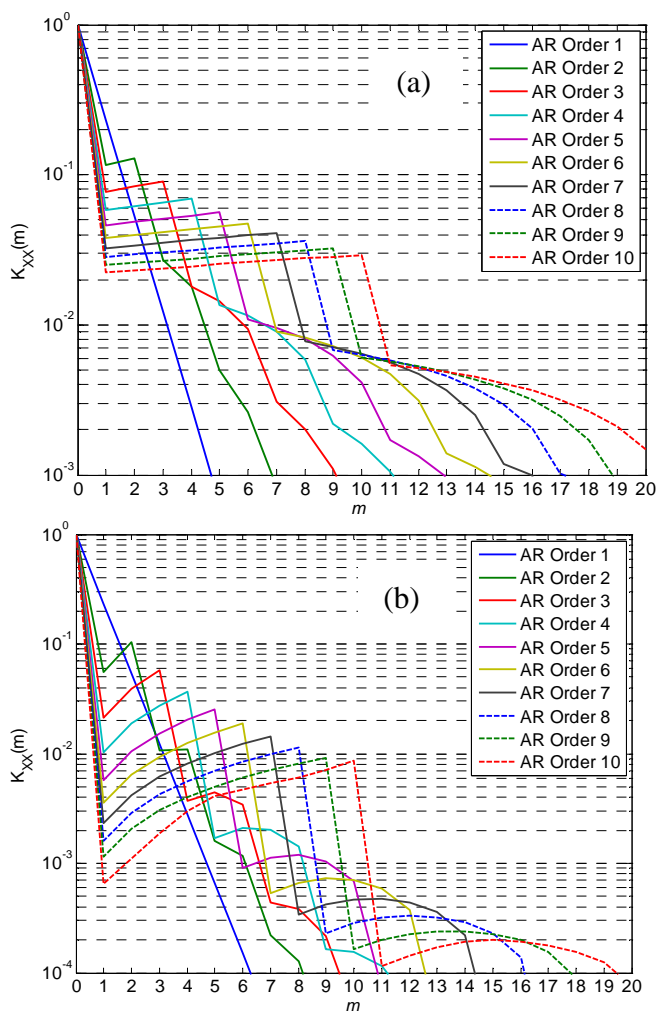

Figure 2. Autocovariance functions obtained for $\sigma_{X}=1$, autoregressive orders. (a) $\bar{L}_{0}=0.3$, (b)

$$
\bar{L}_{1}=0.3 \text {. }
$$

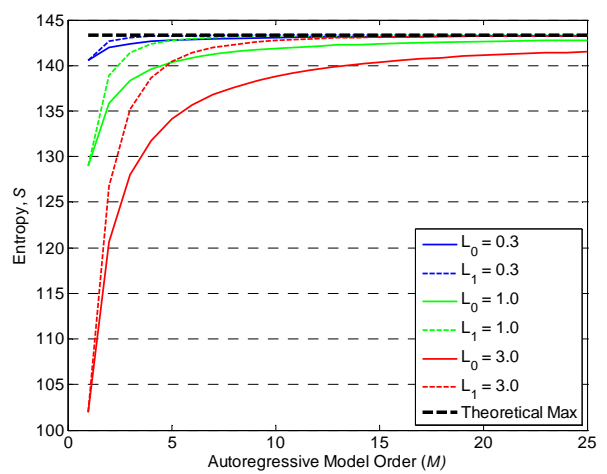

Figure 3. Entropy of the generated process for different correlation length values and definitions.

the respective values of the correlation length $\bar{L}_{0}$ or $\bar{L}_{1}$ obtained from the optimum 
autoregressive coefficients $\hat{a}_{q}$. For a first order autoregressive model, $\bar{L}_{0}$ and $\overline{L_{1}}$ are equal, both to $\left|\hat{a}_{1}\right| /\left(1-\left|\hat{a}_{1}\right|\right)$, but they differ for higher order models. When $\bar{L}_{0}$ is held constant, $\bar{L}_{1}$ increases as a function of the autoregressive order $M$ while $\bar{L}_{0}$ decreases with this order when $\bar{L}_{1}$ is constrained in the optimization, see Fig. 4. While the two correlation length definitions are different, they are metrics of the same property and thus one can expect that they would not be dramatically different from one another. Then, the selection of the autoregressive order can result from a refinement of the optimization process in which a particular value of one correlation length is imposed and a range of values for the other specified, i.e., $\bar{L}_{0}=0.3$ and $\bar{L}_{1} \leq 1$ leads from Fig. 4 to an autoregressive order of $M=6$. Autocovariance functions obtained for various sets of correlation lengths are shown in Figs 5.

Note that the autoregressive model of Eq. (39) not only provides a parametric representation of the autocovariance function but it also provides a simulation algorithm of the values of the process $X_{n}=Z_{n}+\mu_{X}$ as long as $M$ initial values of $Z_{n}$ are available. One approach to generate such initial conditions is to initiate the recursion with deterministic values, $z_{n}=0$ say, for $n=1,2$, ..., $M$ and then march it until stationarity is reached. Note that the length of the transient can be determined from the poles $v$ of the autoregressive model, see Eq. (40). Another approach is to simulate the initial conditions $Z_{n}$ directly as zero mean Gaussian random variables with covariance matrix $\hat{K}_{\underline{Z} \underline{Z}}$ determined to the autocovariance function $K_{Z Z}(m), m=0, \ldots M-1$.

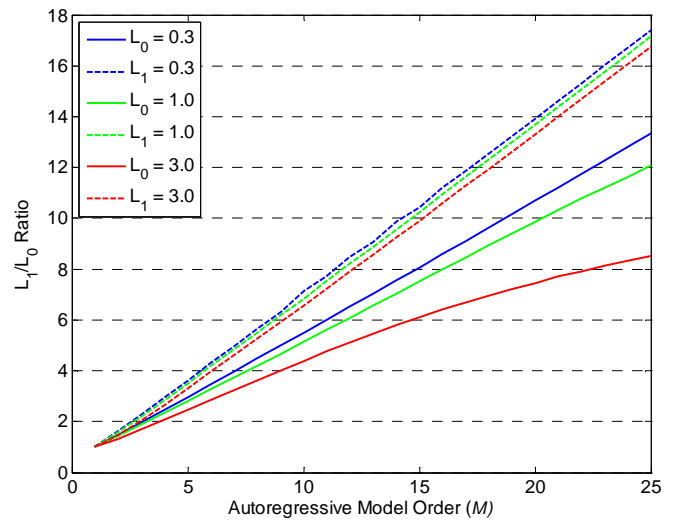

Figure 4. Achieved $L_{1} / L_{0}$ as a function of the autoregressive order for different $\bar{L}_{0}$ and $\bar{L}_{1}$.

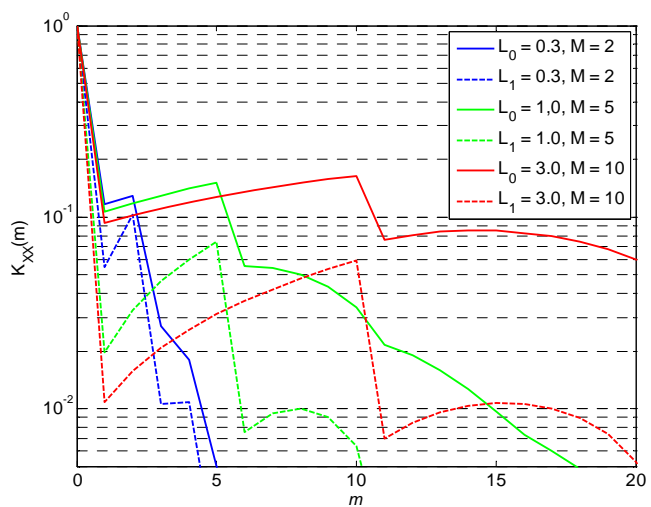

Figure 5. Autocovariance function for some typical conditions.

\subsection{Positive Processes}

Many physical properties are positive and thus the modeling of their uncertainty must maintain this constraint. In the present formulation, this is achieved by restricting the process to exhibit only positive values, i.e., with $\Omega$ being the domain of Eq. (3). In this case, Eq. (25) is a truncated Gaussian distribution and, as such, does not vanish when any of the variable $x_{n}$ goes to zero. This feature of the distribution is not always appropriate, e.g., see Soize (2000). The work carried out here is thus limited to properties which are not required to be strictly positive. 
A result of the truncation of the Gaussian distribution is the lack of closed form expressions for the normalization constant, mean, and covariance matrix, i.e., Eqs (31)-(32) are no longer applicable. These quantities will thus have to be estimated from simulated values of the process $X_{n}$.

These values will be obtained by rejection from the Gaussian process $Y_{n}$, defined as in Eq. (21) but over the entire space, i.e., with $\Omega$ defined by Eq. (2). While this vector could be simulated according to the strategy of Eq. (38), this would lead to an expensive rejection process as any simulated vector $y$ with at least one negative value would be discarded.

A more convenient approach is to proceed recursively with an autoregressive modeling as in Eq. (39). Specifically, let $y_{n}=z_{n}+\mu$ be the value of $Y_{n}$ obtained with a particular sample $w_{n}^{(1)}$ of the Gaussian white noise process $W_{n}$. If $y_{n}$ is positive, it is an acceptable value of $x_{n}$ and thus the assignment $x_{n}=y_{n}$ is performed. In the negative, another value $w_{n}^{(2)}$ of $W_{n}$ is simulated and the corresponding $y_{n}$ is checked for positiveness. The process is then repeated until a positive $y_{n}$ is obtained and the assignment $x_{n}=y_{n}$ performed. The simulation then moves to sample $n+1$.

Two issues arising in the above simulation strategy are: (i) the need for $M$ initial values of $Z_{n}$ and (ii) the required stationarity of the simulated samples. Both can be addressed by starting with deterministic initial conditions, $z_{n}=0$ say, for $n=1,2, \ldots, M$ and then marching the simulation until stationarity is reached. This is achieved for the sample $r$ such that $\left|v_{\max }\right|^{r} \approx 0$ where $v_{\max }$ is the pole of the autoregressive model, see Eq. (40), of largest magnitude. The simulation of stationary values $x_{n}$ then proceeds from sample $r+1$.

The efficiency of the above simulation algorithm adds to the benefits, e.g., smoothness of autocovariance function, of an autoregressive parametrization of the process (as in section 2.2) vs. a direct estimation of its autocovariance function (as in section 2.3). Accordingly, the former approach is presented first below.

\subsubsection{Positive processes - Autoregressive} Modeling

Within this framework, the autoregressive coefficients $\hat{a}_{q}$ and $\hat{b}_{0}$ and the parameter $\mu$ will be selected to maximize the entropy while satisfying (within the accuracy limit of the finite number of samples simulated) the constraints of Eqs (6)-(9). To this end, note from Eqs (1) and (25) that the entropy is $S=-\ln C+\frac{P}{2}+[\underline{\mu} \underline{X}-\underline{\mu}]^{T} G[\underline{\mu} \underline{X}-\underline{\mu}]$

with $\underline{\mu}=\mu \underline{E}, \underline{\mu}_{X}=\mu_{X} \underline{E}$, and $G=\left[\hat{K}_{\underline{X}} \underline{X}\right]^{-1}$. Further, the constant $C$ is expressed as

$$
C=\sqrt{\frac{\operatorname{det}(G)}{(2 \pi)^{P}}} \frac{1}{p_{a c c}}
$$

where $p_{a c c}$ is the probability that a set of $P$ samples $Y_{n}=Z_{n}+\mu$ be all positive. In the autoregressive-based algorithm, this probability can also be evaluated using conditional probabilities. Specifically,

$$
\begin{aligned}
p_{a c c} & =\operatorname{Prob}\left[\underline{Y} \in \mathrm{R}^{+P}\right] \\
& =\prod_{s=1}^{P} \operatorname{Prob}\left[Y_{s} \in \mathrm{R}^{+} \mid \underline{Y}_{s-1} \in \mathrm{R}^{+(s-1)}\right] \\
& =\prod_{s=1}^{P} p_{a c c}^{(s)}=\left[\bar{p}_{a c c}\right]^{P}
\end{aligned}
$$

where $\underline{Y}_{s}$ denotes the vector of components equal to $Y_{n}, n=1, \ldots, s$, and $p_{a c c}^{(s)}$ is the probability of accepting the sample $s$ when computed from the previous ones. Owing to 
the stationarity of the process $Y_{n}$, the probability of acceptance $p_{a c c}^{(s)}$ of a particular sample $s$ is constant, denoted as $\bar{p}_{a c c}$.

The above scheme was exemplified with the correlation length definition of Eq. (8) and $\bar{L}_{0}$ $=0.3,1$, and 3 with means of 2 and 3 . The resulting autocovariance functions of the positive processes were found to be very close, for equal values of the autoregressive orders, to their counterparts for processes without sign constraint of section 2.3, see Fig. 6 as an example.

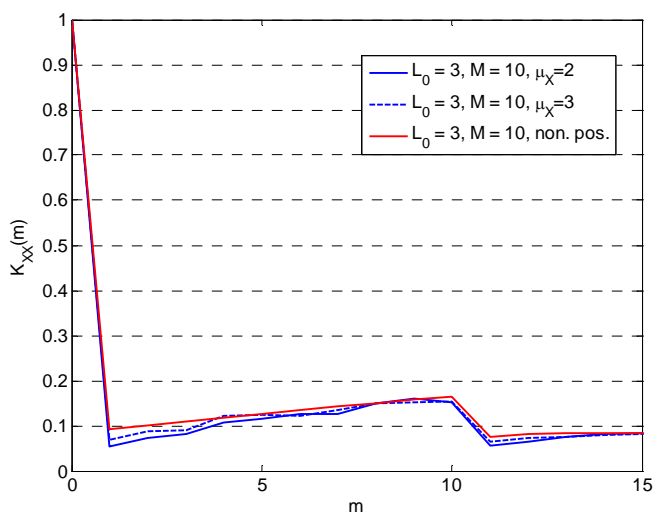

Figure 6. Autocovariance functions of the process without sign constraint ("non-pos.”) and positive process, $\bar{L}_{0}=3$, autoregressive order $M=10$.

2.4.2 Positive processes - Direct Estimation of the Autocovariance Function

The direct estimation approach focuses on the determination of the values of the parameters $\mu, \lambda_{3}, s_{m}$, and $\delta$ to satisfy the mean, Eq. (6), variance, Eq. (7), correlation length, Eq. (11), and sign sequence, Eq. (12), conditions as in section 2.2. The main difference with that earlier section is that the mean and autocovariance function were here determined from a statistical analysis of samples of the process $X_{n}$.

These values were generated using the autoregressive-based simulation with rejection strategy described above. The corresponding coefficients $\hat{a}_{q}$ and $\hat{b}_{0}$ were estimated from the Yule-Walker equations, Eq. (41) for $s=0$, ..., $M=P$, using the autocovariance sequence $K_{Z Z}(m)$ obtained by inversion of the corresponding matrix $G$ as in Eqs (31) and (35).

\section{Friction Modeling in Stick-Slip and Microslip Response}

One objective of the present study was to investigate the effects of uncertainty in friction coefficients on the dynamic response of systems exhibiting stick-slip and microslip behavior. The methodology developed in the previous sections provides the modeling of the uncertain friction coefficients. To demonstrate its application, consider the 5 degree of freedom system shown in Fig. 7 in which each degree of freedom is connected to ground through a slider/friction (Jenkins) element which slides when the force in the corresponding spring is larger than the force of friction.

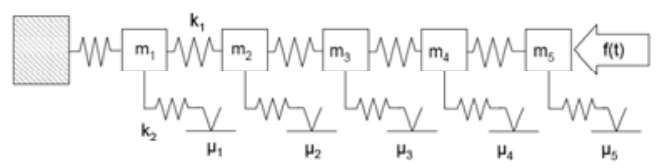

Figure 7. Chain of oscillators with Jenkins elements.

The parameters of each oscillator were selected to be identical, i.e., the masses were chosen as $m_{i}=m=1$ while the stiffnesses $k_{1}$ and $k_{2}$ were selected as 25 and 50, respectively. In regards to the friction, the value of the normal force $N$ was assumed to equal 10 and the static and kinematic coefficients of friction were taken equal, i.e., $\mu_{S}^{(i)}=\mu_{D}^{(i)}$ for each oscillator $i=1, \ldots, 5$. Further, the mean model of the system was assumed to have coefficients of friction $\mu_{S}^{(i)}=\mu_{D}^{(i)}=0.2$ for each oscillator $i=1, \ldots, 5$. In addition, the stuck system was assumed to 
also exhibit a classical viscous damping with a modal damping ratio of $0.5 \%$ on all 5 modes.

Note that the consideration of viscous damping in addition to friction is necessary as the latter does not guarantee alone the finiteness of the response (e.g., see discussion of Den Hartog, 1931). However, the viscous damping is sufficient, i.e., even without friction, to ensure this finiteness condition. Then, the strict positiveness of the simulated coefficients of friction is not required and thus the uncertainty modeling approach described in the previous section is applicable.

The system was subjected to a concentrated force at its free end varying in time as a Gaussian white noise process in the range of $[0,2.5] \mathrm{Hz}$ with a specified variance (see below). The response of the system was obtained through a Newmark- $\beta$ integration scheme with a standard time step of 0.00843 for 400,000 such time steps. Particular care was exercised to accurately pinpoint the transitions of the degrees of freedom from slip to stick and stick to slip. The transition capturing was accomplished by a successive halving of the time steps when a transition was detected until the step was $2^{15}$ time smaller than the standard time step. At that point, transition was assumed to be at the beginning or the end of this small interval depending on the closeness of the transition from these end points. The validation of the algorithm was accomplished by tracking various transitions.

For these parameter values, it was desired next to select a value of the excitation variance that would provide stick-slip of the various masses to exemplify microslip. For low values of the variance, the system remains stuck and the response is linear. As the variance of the excitation is increased, the system slowly changes from fully stuck to fully slipping and the linear natural frequencies of these two extreme conditions are given in Table 1 . For intermediate values of the variance, the desired stick-slip behavior is achieved but differently for each oscillator.
Specifically, the fraction of time spent in slip mode decreases monotonically from the degree of freedom 5 on which the force is applied to the first one, nearest to the wall, see Table 2 for the two variances of excitation considered here: 3.7 and 41.1 .

The physics described above is reflected in the power spectra of the stationary responses shown in Figs 8. For the lower excitation case, Fig. 8(a), the responses of the various degrees of freedom exhibit clear peaks at the stick natural frequencies of Table 1 . For the first degree of freedom (nearest to the wall), the energy is concentrated in the band of the natural frequencies consistently with a nearly linear system. Proceeding toward the free end, the degrees of freedom exhibit an increased percentage of time in slip, i.e., an increased nonlinearity in response, which is seen in Fig. 8(a) to induce a transfer of energy out of band: the increase in variance of the response is not significantly reflected by an increase in peak responses but rather in the spectrum outside of the band of natural frequencies. For the higher excitation case, Fig. 8(b), the linear peaks have completely

Table 1. Natural frequencies $(\mathrm{Hz})$ of the system in slip and stuck modes

\begin{tabular}{cccccc}
\hline \multirow{2}{*}{ Mode } & \multicolumn{5}{c}{ Natural Frequencies (Hz) } \\
\cline { 2 - 6 } & 1 & 2 & 3 & 4 & 5 \\
\hline Stuck & 1.15 & 1.31 & 1.53 & 1.75 & 1.90 \\
\hline Slip & 0.23 & 0.66 & 1.04 & 1.34 & 1.53 \\
\hline
\end{tabular}

Table 2. Percentage of time spent in slip mode for each oscillator for the two variances of the excitation.

\begin{tabular}{cccccc}
\hline \multirow{2}{*}{ Variance } & \multicolumn{5}{c}{ Oscillator } \\
\cline { 2 - 6 } & 1 & 2 & 3 & 4 & 5 \\
\hline 3.7 & $7 \%$ & $8 \%$ & $12 \%$ & $22 \%$ & $37 \%$ \\
\hline 41.1 & $36 \%$ & $45 \%$ & $52 \%$ & $59 \%$ & $65 \%$ \\
\hline
\end{tabular}



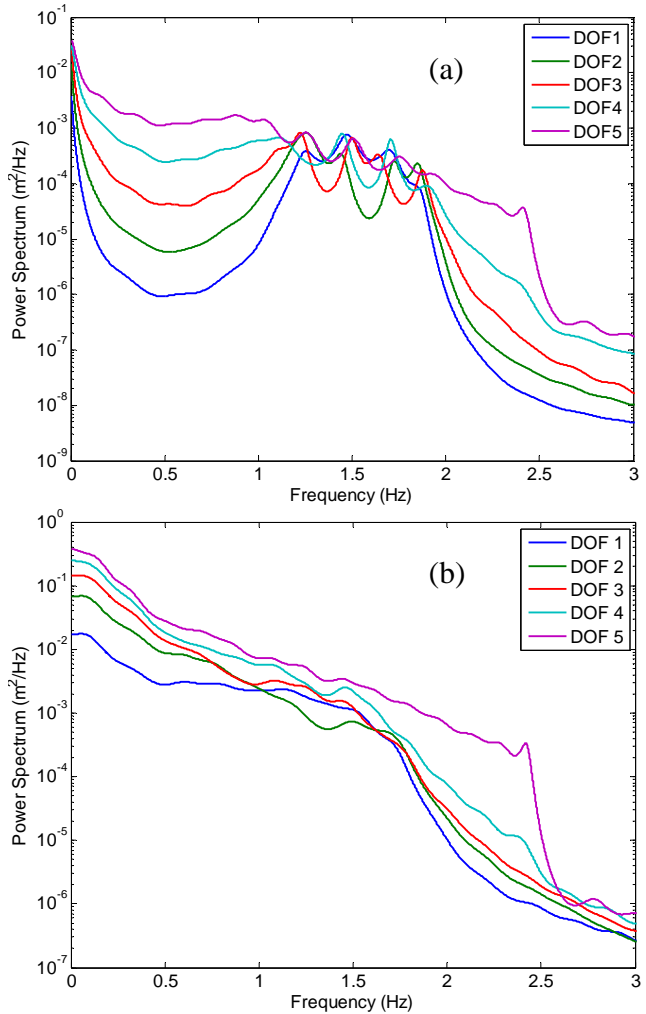

Figure 8. Power spectral densities of the response of the 5 different oscillators, no uncertainty.

Excitation variance (a) 3.7, (b) 41.1.

disappeared and energy is quite broadly distributed with only weakly seen peaks at the frequencies of the system in slip.

Uncertainty in the coefficients of friction was next introduced as described in the first part of this paper. Two particular levels of variability of these coefficients were considered, i.e., standard deviations of 0.067 and 0.10 , with the mean maintained at the value of 0.2 . Three correlation lengths $L_{0}=$ $0.3,1.0$, and 3.0 were achieved with autoregressive models of order 2, 5, and 10, respectively. The responses of the system with these 6 combinations of coefficients of friction uncertainty were computed for 100 samples each for both force variances of 3.7 and 41.1. Of particular interest here are the variations of the power spectra of the responses and of the fraction of time in slip resulting from the uncertainty in friction coefficients. The latter characteristic was measured by the standard deviation of the fraction of time in slip for each degree of freedom. It is plotted vs. its mean in Figs 9 for the two levels of uncertainty on the coefficients of friction. Even though each of these plots includes data from different degrees of freedom and different excitation levels for the three different correlation lengths, a clear pattern is seen that the largest variability in the fraction of time in slip occurs for oscillators that are approximately $30 \%$ of the time in slip in the mean. This observation seems consistent with the data of Table 1 and Figs 8 , i.e., the strongest qualitative changes in the response occur for the degrees of freedom exhibiting this range of fractions of time in slip. Note further that the increase in standard deviation of the coefficients of friction from 0.067 to 0.1 leads to a smaller increase in the standard deviation of the fraction of time in slip. Finally, no consistent effect of the correlation length is seen although it appears on Fig. 9(b) that the correlation length $L_{0}=1$ typically yields larger standard deviations of fraction of time in slip than $L_{0}=3.0$ and in turn larger than those for $L_{0}=0.3$.

Consider next the variability of the power spectra which is tracked here by their 5th and 95th percentiles computed at each frequency. For clarify, the range of responses between these percentiles is referred to here as the band of uncertainty. Shown in Figs 10 are representative bands of uncertainties obtained for different cases. To provide a more condensed perspective on the effects of the correlation length and standard deviation of the coefficients of correlation, the curves shown in Figs. 10, i.e., 5th and 95th percentiles and mean of the random spectra, were reduced to pseudo-variances by integration over the entire frequency domain, 


$$
\sigma_{95,5, m}^{(i) 2}=\int_{-\omega_{b}}^{\omega_{b}} S_{95,5, m}^{(i)}(\omega) d \omega
$$

where $S_{95,5, m}^{(i)}(\omega)$ denotes the 95th percentile, 5th percentile, and mean of the random spectra of degree of freedom $i$. Further, $\omega_{b}=120 \pi$ is the Nyquist frequency. Then, the width of a particular uncertainty band was measured by the ratio of the pseudovariances corresponding to the 95th and 5th percentiles,
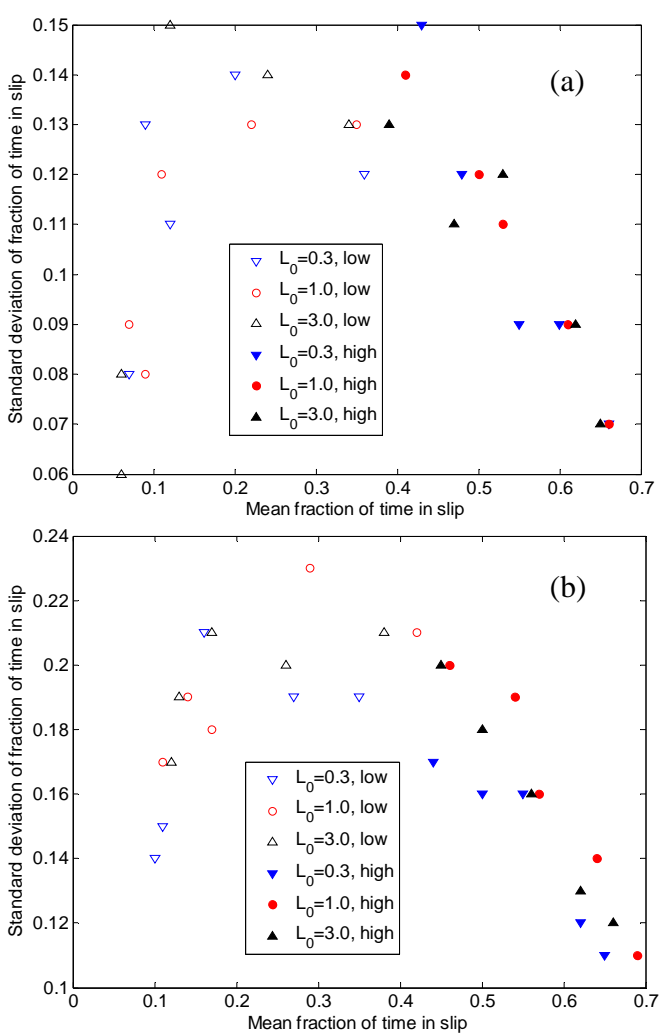

Figure 9. Standard deviation of the fraction of time in slip vs. its mean for the various correlation lengths, for all oscillators, and both force variances ("low" and "high" corresponding to 3.7 and 41.1), standard deviation of coefficients of friction of (a) 0.067, (b) 0.1 .
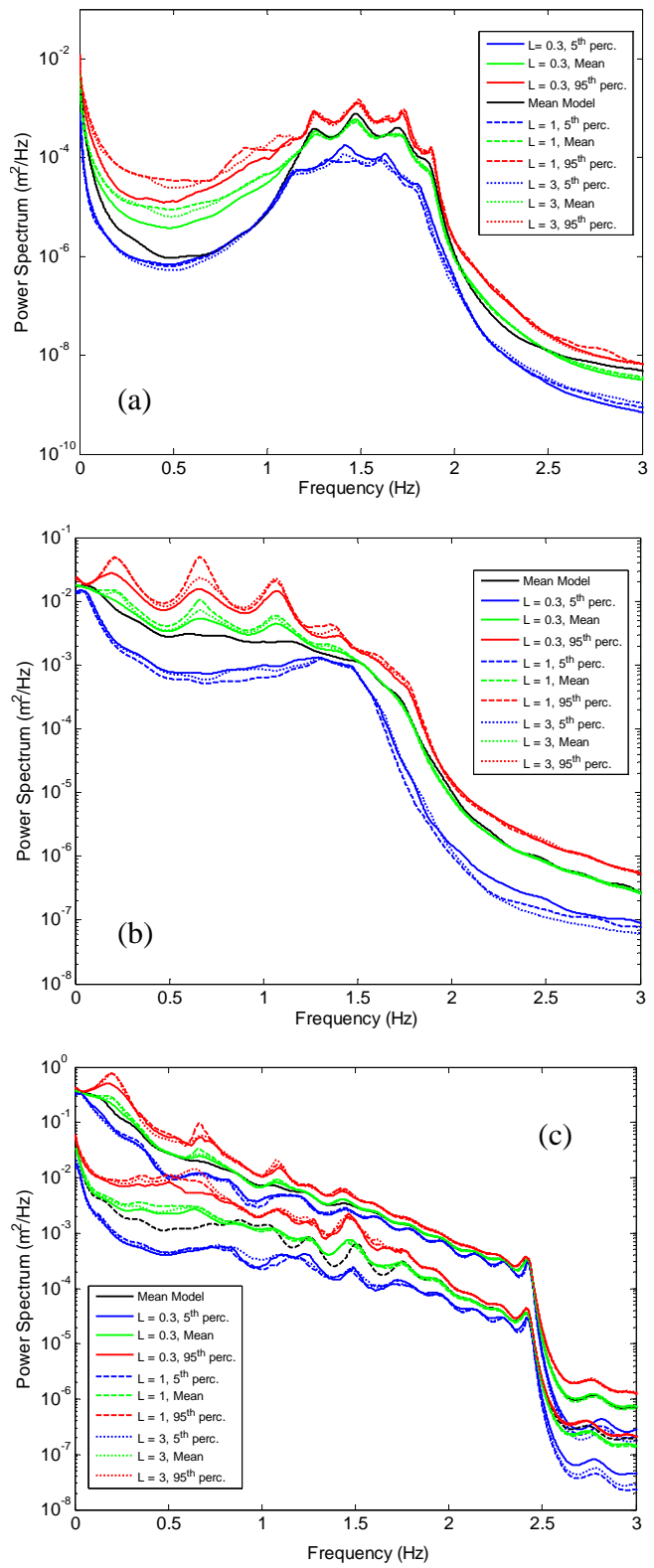

Figure 10. Power spectra of the response of degree of freedom (a),(b) 1 and (c) 5, mean, 5th, and 95th percentiles, for various correlation lengths $L_{0}$, standard deviation of coefficients of friction 0.10 Excitation variance (a) 3.7 (b) 41.1, (c) 3.7 (lower set of curves) and 41.1 (higher set of curves). 
i.e., $\sigma_{95}^{(i) 2} / \sigma_{5}^{(i) 2}$. In light of the observations drawn on the basis of Figs 9, it is of particular interest to plot this ratio as a function of the mean fraction of time in slip, see Figs 11.

The detailed analysis of Figs $10-11$ revealed foremost that:

(i) the width of the band of uncertainty on the spectrum of a particular oscillator appears to be a monotonically decreasing function of the fraction of time the associated slider slips, see Figs 11 . Thus, the bands of uncertainty tend to decrease as the excitation level is increased since that promotes increasing slip, e.g., compare Figs 10(a) and 10(b), and the two sets of curves of Fig. 10(c).

(ii) the correlation length has a definite effect on the width of the bands of uncertainty, see Figs 11. This effect appears to be complex for lower mean fractions of time in slip but more clear at higher fractions with the maximum width occurring at an intermediate correlation length ( $L_{0}=1$ here) for the larger uncertainty level and at the longest correlation $\left(L_{0}=3\right.$ here) for the lower uncertainty level.

Additionally, it was found that an increase in the level of uncertainty on the coefficients of friction does not always lead to an increase in the 95th percentile of the spectrum of the response. This is particularly so for oscillators which mostly stick, e.g., degree of freedom 1 at lower excitation level, for which slip creates dissipation and an energy transfer out of band as seen in Fig. 8. Further, it was observed that the mean of the random spectra is closer to the 5th percentile than to the 95th one suggesting that the distribution of the spectral values at given frequencies exhibit a longer tail for larger values. Finally, it is interesting to observe the presence of significant peaks on the 95th percentiles of the spectra of oscillators exhibiting a significant slip, e.g., see Figs. 10(b) and 10(c), which are not readily seen on the corresponding spectra in the absence of uncertainty, Figs (8). These peaks appear to closely match the natural frequencies of the system in slip which suggests that certain combinations of friction coefficients can promote the energy transfer through the system at these frequencies.
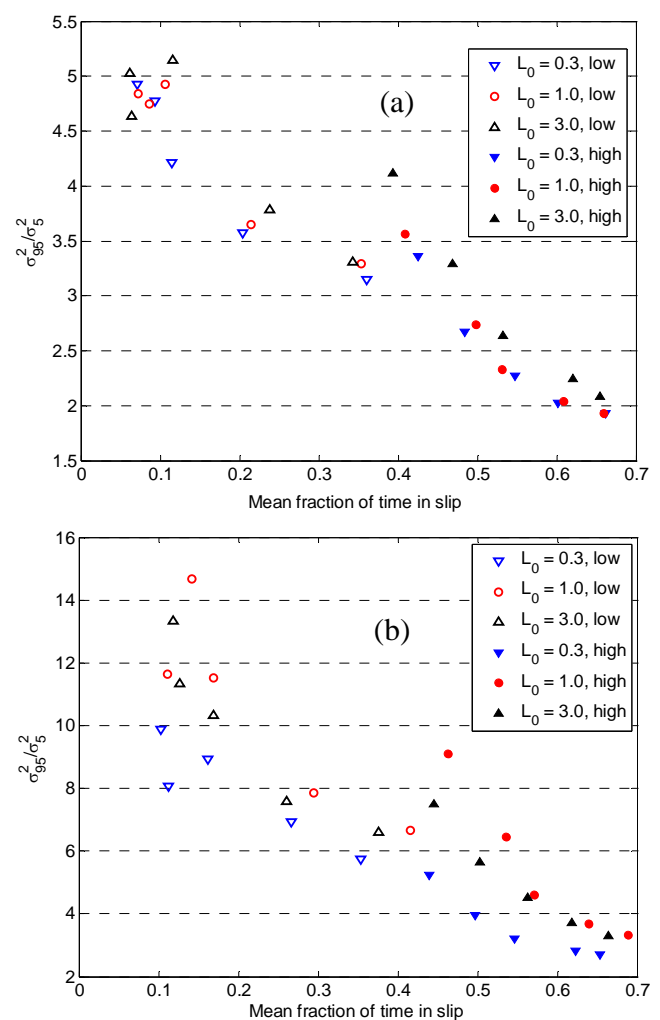

Figure 11. Ratio of the pseudo-variances corresponding to the 95th and 5th percentiles vs. mean fraction of time in slip for the various correlation lengths, for all oscillators, and both force variances ("low" and "high" corresponding to 3.7 and 41.1), standard deviation of coefficients of friction of (a) 0.067, (b) 0.1.

\section{Summary}

The focus of this investigation has been on the formulation and a first application of a novel model for the representation of uncertain properties as discrete stationary random processes. As opposed to postulating the distribution of the random process values, this function is here derived to yield the maximum of the entropy under the set of constraints 
representing a given mean, a given variance, and a given correlation length. In the absence of a sign constraint on the process, its distribution is Gaussian with autocovariance function that is expressed in terms of the Lagrange multipliers of the constraints. Two numerical approaches were described and demonstrated for the evaluation of this autocovariance function and its near white noise features were discussed.

For positive processes, the distribution obtained is a truncated Gaussian and the above computational strategies were extended to permit the estimation of the autocovariance function of the process and the efficient simulation of its realizations. Results obtained with different correlation lengths and coefficients of variation suggest that the autocovariance function of the positive process is very close to its equivalent for the process without sign constraint.

The novel model proposed here was next applied to the simulation of uncertain coefficients of friction in a chain of oscillator with Jenkins elements representing the dynamic response of a flexible structure connecting through friction to a rigid foundation. This model permits the consideration of microslip of the structure. The introduction of uncertainty on the coefficients of friction was found to have notable effects on the response which typically correlate to the mean fraction of time in slip of the slider attached to a particular degree of freedom. Three key observations were that:

(i) the standard deviation of the fraction of time in slip exhibited a peak at an intermediate value (about 0.3 here) of the mean value of this fraction,

(ii) the width of the uncertainty band on the spectrum of the response of a particular oscillator was a decreasing function of the mean fraction of time in slip,

(iii) the coefficient of correlation has a definite effect on the width of the bands of uncertainty which appears to be complex for lower mean fractions of time in slip but more clear at higher fractions with the maximum width occurring at an intermediate/higher correlation lengths depending on the level of uncertainty on the coefficients of friction.

\section{Acknowledgments}

The partial support of this work through the Oversea Research Program of GNU and 2nd phase of BK21 is gratefully acknowledged.

\section{References}

Berger, E. J. and Deshmukh, D. V., Convergence Behaviors of Reduced-Order Models For Friction Contacts, Journal of Vibration and Acoustics, 127, 370-381, 2005.

Den Hartog J. P., Forced Vibrations With Combined Coulomb and Viscous Friction, Transactions of the American Society of Mechanical Engineers, 53(9), 107-115, 1931.

Deshmukh, D. V., Berger, E. J., Begley, M. R. and Komaragiri, U., Correlation of a Discrete Friction (Iwan) Element and Continuum Approaches to Predict Interface Sliding Behavior, European Journal of Mechanics A/Solids, 26, 212-224, 2007.

Ghanem, R. and Spanos, P. D., Stochastic Finite Elements: A Spectral Approach, Spring-Verlag, New York, 1991.

Mignolet, M. P. and Spanos, P. D., Recursive Simulation of Stationary Multivariate Random Processes. Part I, Journal of Applied Mechanics, 109, 674-680, Sept, 1987.

Nigam, N. C., Introduction to Random Vibrations, MIT Press, 1983.

Sachdeva, S.K., Nair, P.B. and Keane, A.J., On using Deterministic FEA Software to Solve Problems in Stochastic Structural Mechanics, Computers and Structures, 85, 277-290, 2007.

Schueller, G. I., On the Treatment of Uncertainties in Structural Mechanics and Analysis, Computers and Structures, 85, 235-243, 2007.

Segalman, D. J., Gregory, D. L., Starr, M. J., Resor, B. R., Jew, M. D., Lauffer, J. P. and Ames, N. M., Handbook on Dynamics of Jointed Structures, Sandia Report SAND2009-4164, 2009.

Sinha, A. and Griffin, J. H., Effects of Friction Dampers on Aerodynamically Unstable Rotor Stages, AIAA Journal, 23( 2), 262-270, 1985. 
Soize, C., A Nonparametric Model of Random Uncertainties on Reduced Matrix Model in Structural Dynamics, Probabilistic Engineering Mechanics, 15(3), 277-294, 2000.

Soize, C., Maximum Entropy Approach for Modeling Random Uncertainties in Transient Elastodynamics, Journal of the Acoustical Society of America, 109(5), 1979-1996, 2001.
Soize, C. Non-Gaussian Positive-Definite MatrixValued Random Fields for Elliptic Stochastic Partial Differential Operators, Computer Methods in Applied Mechanics and Engineering, 195, 26-64, 2006.

Soize, C., Stochastic Models of Uncertainties in Computational Mechanics, American Society of Civil Engineers (ASCE), Reston, 2012. 